\title{
Evaluation of Exposure Risk in the Weaving Process of MWCNT-Coated Yarn with Real-time Particle Concentration Measurements and Characterization of Dust Particles
}

\author{
Mitsutoshi TAKAYA ${ }^{1}$, Mariko ONO-OGASAWARA ${ }^{1}$, Yasushi SHINOHARA, \\ Hisayo KUBOTA ${ }^{1}$, Shuji TSURUOKA ${ }^{2}$ and Shigeki KODA ${ }^{1}$ \\ ${ }^{1}$ National Institute of Occupational Safety and Health, Japan, 6-21-1, Nagao, Tama-ku, Kawasaki, Kanagawa \\ 214-8585, Japan \\ ${ }^{2}$ Research Center for Exotic NanoCarbons, Shinshu University, 4-17-1, Wakasato, Nagano 380-8553, Japan
}

Received July 13, 2011 and accepted November 28, 2011

Published online in J-STAGE February 1, 2011

\begin{abstract}
Various applications of multiwalled carbon nanotubes (MWCNT) have been developed. One of these applications is an efficient sheet heating element that is woven from MWCNT-coated yarn. In this research, we assessed the exposure to MWCNT and/or the probability of particle release from broken MWCNT-coated yarn during the weaving process. This was accomplished using particle concentrations, microscopic observation, and carbon analysis. In the weaving process, neither an increase in the number of particles nor a difference in particle-size distribution was observed. In the scanning electron micrographic observation, nanosize MWCNT particles were not detected, but there were micron-size particles containing MWCNT as fragments of the yarn. Carbon analysis showed the concentration of micron-size particles containing MWCNT did not exceed $0.0053 \mathrm{mg}-\mathrm{C} / \mathrm{m}^{3}$ around the loom. This value was much lower than the respirable dust mass concentration. Most of micron-size particles seemed to originate from polyester yarn without MWCNT coating. It is recommended that workers use conventional (even not specialized for nanoparticles) personal protective equipment such as respirators and gloves to prevent exposure to respirable-size MWCNT-containing particles. The probability of MWCNT fall-off from the MWCNT-coated yarn was not detected by transmission electron microscopic observation of MWCNT-coated yarn before or after the weaving process.
\end{abstract}

Key words: Multiwalled carbon nanotube, MWCNT, Fabrication, EC, SEM, TEM

\section{Introduction}

Multiwalled carbon nanotubes (MWCNT) are a new material consisting of multiple concentric layers of cylindrical graphene sheets. Industrial production of MWCNT

*To whom correspondence should be addressed.

E-mail: takaya@h.jniosh.go.jp

(C)2012 National Institute of Occupational Safety and Health was developed in Japan. The diameter of MWCNT, depending on the number of layers, range from 10-100 $\mathrm{nm}$ and their lengths from 100-10,000 nm. MWCNTs are categorized as so-called "nanomaterials". MWCNT is a promising material because it demonstrates specific electrical conduction properties and physical strength. A wide variety of other applications are being developed and one of the practical applications has already been achieved, 
which was to improve the properties of a secondary cell by mixing it with MWCNT. Other applications are being developed though the addition of MWCNT to paper, fibers, ceramics, and so on.

In this research exposure measurements were conducted at the workplace where yarns coated with MWCNT (MWCNT-coated yarn) were woven to produce a conductive fabric. This MWCNT-coated yarn, named CNTEC, was developed by Kuraray Living Co., Ltd. and Mitsui Co. Ltd. ${ }^{1,2}$. They use a new dispersion technology, which enables MWCNT agglomerates to dissociate into single MWCNTs in water suspension, and the MWCNT-coated yarns are produced by coating polyester yarns with this suspension. The MWCNT-coated yarns are woven into efficient fabric heaters. Tests for practical use have revealed that they work well at preventing freezing and show more homogeneous heat generation than conventional sheet heating elements ${ }^{3)}$. Product stewardship has been considered over the life cycle of the MWCNT-coated yarn and products. For example, MWCNT-coated yarns are treated in a way that prevents MWCNT from falling off, the conducting fabrics are delivered to downstream users in a sealed package, and the coated yarns are recycled back to the distributor after use. Therefore, great attention is being paid to avoid release of fabric fragments into the environment during use of the conducting fabric, and to mitigate workers' exposure in the working environment. The background to these control activities is the concern about the potential hazards of MWCNTs.

As with other nanomaterials, the primary particles of MWCNT are very small and even aggregates/agglomerates are often observed as particles smaller than $4 \mu \mathrm{m}$. Therefore, many tests to assess the potential hazards of MWCNT to the respiratory system have been conducted. The Organisation for Economic Cooperation and Development (OECD) is trying to promote the standardization of test methods for MWCNT as well as for other nanomaterials. However, because the health hazards assessed by the test with one particular MWCNT are not considered representative of other MWCNTs, some companies have set their own occupational exposure limits (OEL) by conducting risk assessments of their products ${ }^{4)}$. Some research institutes have conducted preliminary risk assessments of MWCNTs according to their own strategies and have proposed calculated OELs ${ }^{5,6)}$. At present, there is no consensus for assessing the health effects of MWCNTs. In various processes using MWCNTs, control measures are applied to reduce risk as much as is practically possible for workers, and downstream users and consumers according to the precautionary principal.

In this research, particle concentrations in the environment and personal exposures near workers were measured to assess the working environment. This approach is to assess the exposure to MWCNT in a treatment process for MWCNT-containing material for downstream users, not for MWCNT production. There is very little information on MWCNT-quantified exposure during production ${ }^{7}$, packaging $^{8)}$, and weighing ${ }^{9)}$ of MWCNT, and the possible risk to downstream users of the composite ${ }^{10}$. Various measurement methods have been used in these field surveys because there is no specific method for exposure measurement of MWCNTs. An adequate combination of measurement methods should be applied by considering the materials, processes, and environment. In the present study, we tried to quantify MWCNT exposure in the workplace according to our proposed flow ${ }^{11)}$ as follows: 1) Real-time monitoring of particle mass, number, and size distribution, if particles can be detected, 2) qualifying exposure by electron microscopy, and 3) quantifying MWCNT by carbon analysis. In the workplace, because there was dust from polyester yarns and fabric released from other looms in operation, it was difficult to assess exposure to dust from the MWCNT-coated yarn specifically by measuring particle number and mass. Observation by electron microscopy and carbon analysis of suspended particles made it possible to assess the MWCNT-related exposure to some extent. We also assessed individual MWCNT-fiber release from the MWCNT-coated yarn using transmission electron microscopic (TEM) observation.

\section{Material and Methods}

The textiles produced using CNTEC are woven by Matsubun Textile Co. Ltd. in Katsuyama, Fukui. Fukui is one of the most active textile industry regions in Japan, and there are many textile-producing factories that have superior skills at twisting and weaving yarns. Matsubun Textile Co. Ltd. is one of those factories and the main product of the factory is a polyester textile using automatic rapier looms. The weaving process for heating element textiles is the same as that used for usual polyester textiles except for the types of yarns used. The MWCNT-coated yarn is used as the weft, and copper wires are used in a few threads of the warp for the contact electrode.

The field survey of the present study was carried out from July 20-22, 2010. During the field survey, the weather was hot and sunny, and secondary generation of nanosize particles might have occurred. During our investigation in 
the workplace, a loom for weaving the heating element fabric and 20-40 (depending on time) looms for polyester textiles were working. We measured the particle concentration and size distribution in the air near the loom weaving the heating element fabric both with and without the MWCNTcoated yarn and collected samples of airborne particles.

\section{Devices for real-time monitoring and sampling}

Particle concentrations and size distribution: Nanosizeparticle concentrations were measured by a Condensation Particle Counter (CPC, TSI3007: TSI Incorporated, Shoreview, MN, USA) and, submicron-size particle concentrations were measured by an Optical Particle Counter (OPC, KA-80B; RION Co. Ltd., Kokubunji, Tokyo, Japan). The size distributions were measured by a Scanning Mobility Particle Sizer (SMPS, TSI Inc.). These three devices were placed on a mobile cart and were operated simultaneously at the same sampling points in order to compare the data from each instrument. A portable dust monitor (LD-6, Sibata Science Technology Ltd., Soka, Japan) was also used to monitor the dust concentration around the loom.

Airborne particle sampling: The airborne particles were sampled using a Sioutus Cascade Impactor (SCI) (SKC Inc., Eighty Four, PA, USA) ${ }^{12)}$, which can classify particles into five fractions at a flow rate of $91 / \mathrm{min}$. The size of each fraction in terms of their aerodynamic particle diameters was: Stage A, 6,600-2,500 nm; Stage B, 2,500-1,000 nm; Stage C, 1,000-500 nm; Stage D, 500-250 nm; Stage E (back-up stage), $<250 \mathrm{~nm}$. In the present study, an additional collection stage was added above Stage A to eliminate coarse particles. The size of the $50 \%$ cut-point is $6,600 \mathrm{~nm}$ calculated from the diameter of the SCI inlet when the flow rate is $9 \mathrm{l} / \mathrm{min}$. In this study, total particles collected on the five stages from stages A to E of the SCI, smaller than 6,600 $\mathrm{nm}$, were considered a respirable fraction. Two types of collecting media were used, a quartz fiber filter (QMA: Whatman, Maidstone, Kent, UK) for carbon analysis and a polycarbonate membrane filter (Isopore: Millipore, Billerica, MA, USA) for the Scanning Electron Microscope (SEM) observation. Quartz fiber filters were pre-heattreated in order to decrease organic carbon contamination. A personal dust sampler (NWPS-254: Sibata Scientific Technology Ltd.), which can collect coarse particles and particles with dimensions less than 4,000 nm, separately, was used to measure personal exposure dust mass concentrations. The sampling filters $(25 \mathrm{~mm} \phi$, Pallflex T60A20, Pall Corporation, Washington, NY, USA) were weighed before and after use on an electronic balance (UMX-2 Mettler-Toledo International Inc., Columbus, OH, USA) which is in constant temperature and humidity condition and of which reciprocal sensibility was $0.1 \mu \mathrm{g}$.

\section{Sampling design}

Real-time monitoring and particle sampling were conducted at five sampling points. Point (1) (monitoring and sampling) was located approximately $1 \mathrm{~m}$ away from the woven-textile-ejecting side of the loom, on the opposite side of the usual operation position of the operator, height $1.3 \mathrm{~m}$. Point (2) (sampling) was located approximately 0.3 $\mathrm{m}$ from the shed of the loom, height $1 \mathrm{~m}$. Personal sampling was conducted by a person standing near the operator who was working at the loom and walking around the loom during the absence of the operator. SCI was used as the personal sampler. Other sampling points were located outside the facility and in an office in another building. Points (1) and (2) are shown in Fig. 1. Table 1 shows the arrangement of sampling for carbon analysis.

The deposited dusts around the loom were wiped with 1 $\times 1.5 \mathrm{~cm}$ quartz-fiber filters over about a $10 \times 10 \mathrm{~cm}$ area. The filters were analyzed by a carbon aerosol monitor without any additional processing.

\section{Carbon analysis by carbon aerosol monitor}

Carbon analysis was carried out with a Carbon Aerosol Monitor (Sunset Laboratory, Inc., OR, USA) using a thermal-optical method with the IMPROVE protocol ${ }^{13)}$. Elemental carbon (EC) in particles collected on the quartz fiber filters is oxidized to carbon dioxide in a $\mathrm{He} / \mathrm{O}_{2}$ atmosphere, and the carbon dioxide is reduced to methane; then the methane is quantified by an flame ionization detector. Because the EC of different structures is usually oxidized at different temperatures, strongly graphitic MWCNT in suspended particles can be measured separately from EC in ambient particles by selecting a specific oxidizing temperature. Target MWCNT involved in the MWCNTcoated yarn Baytube ${ }^{\circledR}$ (C150P) is thin (diameter less than $20 \mathrm{~nm}$ ) and is oxidized at a lower temperature than thicker MWCNTs. Therefore, it was difficult to separate the MWCNT in the yarn from ambient EC. In this study, the oxidizing temperature was changed to quantitatively identify and semi-quantify the MWCNT. Under ordinary conditions of carbon analysis with the IMPROVE method, graphitic carbon is oxidized at $700^{\circ} \mathrm{C}$ and $850^{\circ} \mathrm{C}$. When MWCNT-coated yarn is oxidized at $700^{\circ} \mathrm{C}$, the polyester yarn and the coated MWCNT are oxidized simultaneously. In this analysis, polyester yarn and ambient EC were mainly oxidized at $600^{\circ} \mathrm{C}$, and the EC left behind and most of the MWCNT could be detected at $920^{\circ} \mathrm{C}$. This EC 


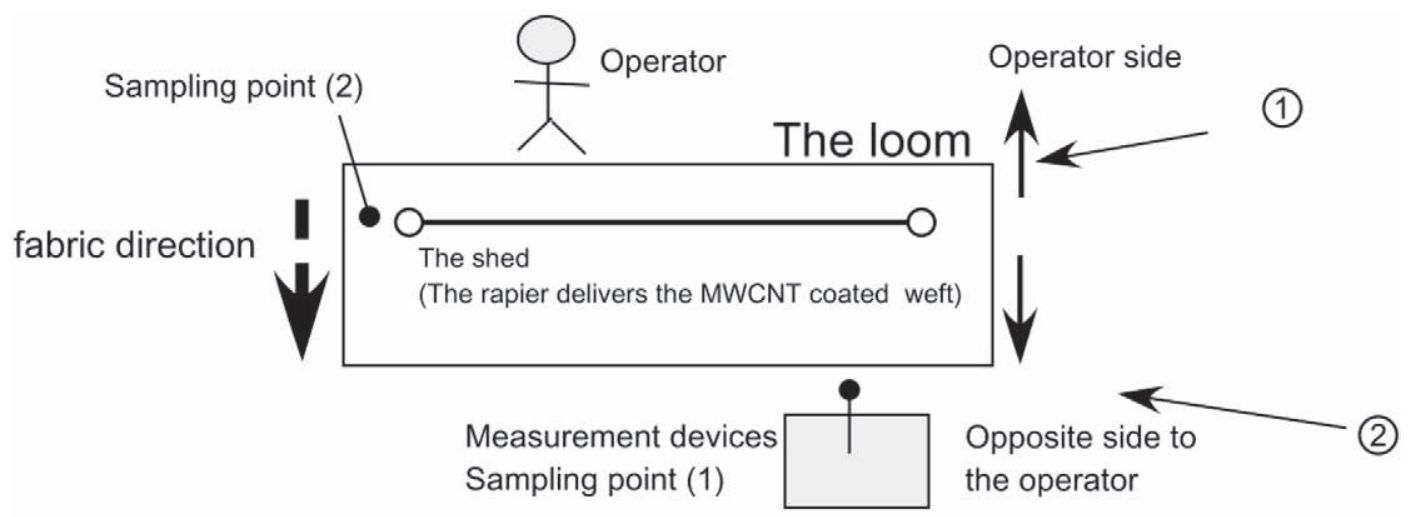

view from (1)

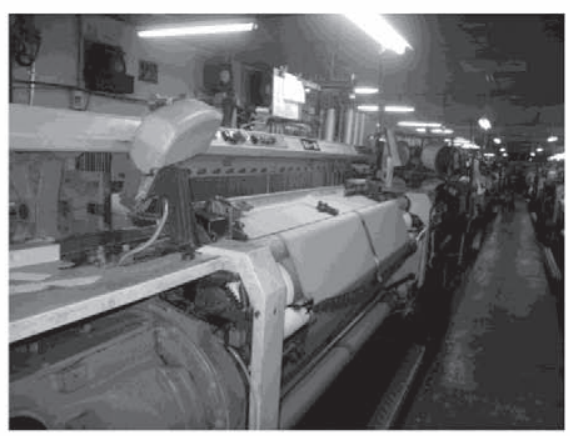

Operator side view from (2)

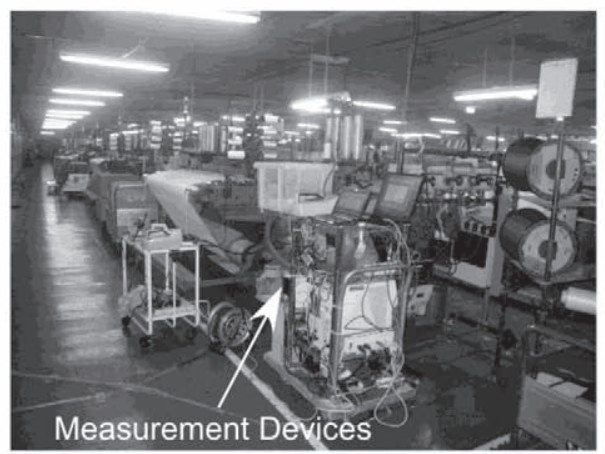

Opposite side to the operator

Fig. 1. Schema of the sampling positions and photographs of around the loom and measurement devices.

Table 1. Sampling points and time

\begin{tabular}{llcc}
\hline Sample name & \multicolumn{1}{c}{ Sampling point } & Date & Time \\
\hline 1 w/o Work & Point (1)* & July 21 & Night \\
1-AM & Point (1)* & July 21 & AM \\
1-PM & Point (1)* & July 21 & PM \\
1 and 2** & Point (1)*+ Point (2)* & July 22 & AM \\
Personal AM & Personal*** & July 21 & AM \\
Personal PM & Personal*** & July 21 & PM
\end{tabular}

*Point (1): $1 \mathrm{~m}$ from the loom; Point (2): on the loom; See Fig. 1. **Sampling duration: 85 min at point (1); 20 min at point (2).

***Sample collected by an author standing near the loom, not the same as a worker's personal exposure.

is called $\mathrm{EC}_{920}$ here. This final temperature was set much higher than it would be with the ordinary method in order to oxidize all the leftover EC which was not oxidized at previous stages. In comparison with the ordinary method, although larger amounts of EC from the polyester yarn and ambient particles not oxidized at $600^{\circ} \mathrm{C}$ can be detected as $\mathrm{EC}_{920}$, the analytical condition was selected on a preferen- tial basis to detect the MWCNT. The quantitation limit of this method at the facility, where background EC is low, is $0.001 \mathrm{mg} / \mathrm{m}^{3}$ for $1 \mathrm{~m}^{3}$ sampling of air.

\section{Particle characterization with Scanning Electron} Microscope (SEM)

The sampled filters were cut into rectangles of approximately $4 \times 2 \mathrm{~mm}$. A cut piece of the filter was fixed to an aluminum sample stage with electro conductive selfadhesive tape. To avoid charge collection problems, the sample was treated platinum-palladium deposition by ionsputter (E-1030, Hitachi Co. Ltd., Tokyo, Japan). The samples were observed using a SEM (S-4700, Hitachi Co. Ltd.). The acceleration voltage was $10 \mathrm{kV}$ and the magnification ratio range was from 30 to 50,000 .

\section{Sample yarn analysis by Transmission Electron Microscopy} (TEM)

A yarn sample of approximately $5 \mathrm{~mm}$ in length was embedded in epoxy resin. The embedded resin block was sliced into $1 \mu \mathrm{m}$-thick sections on a microtome for 

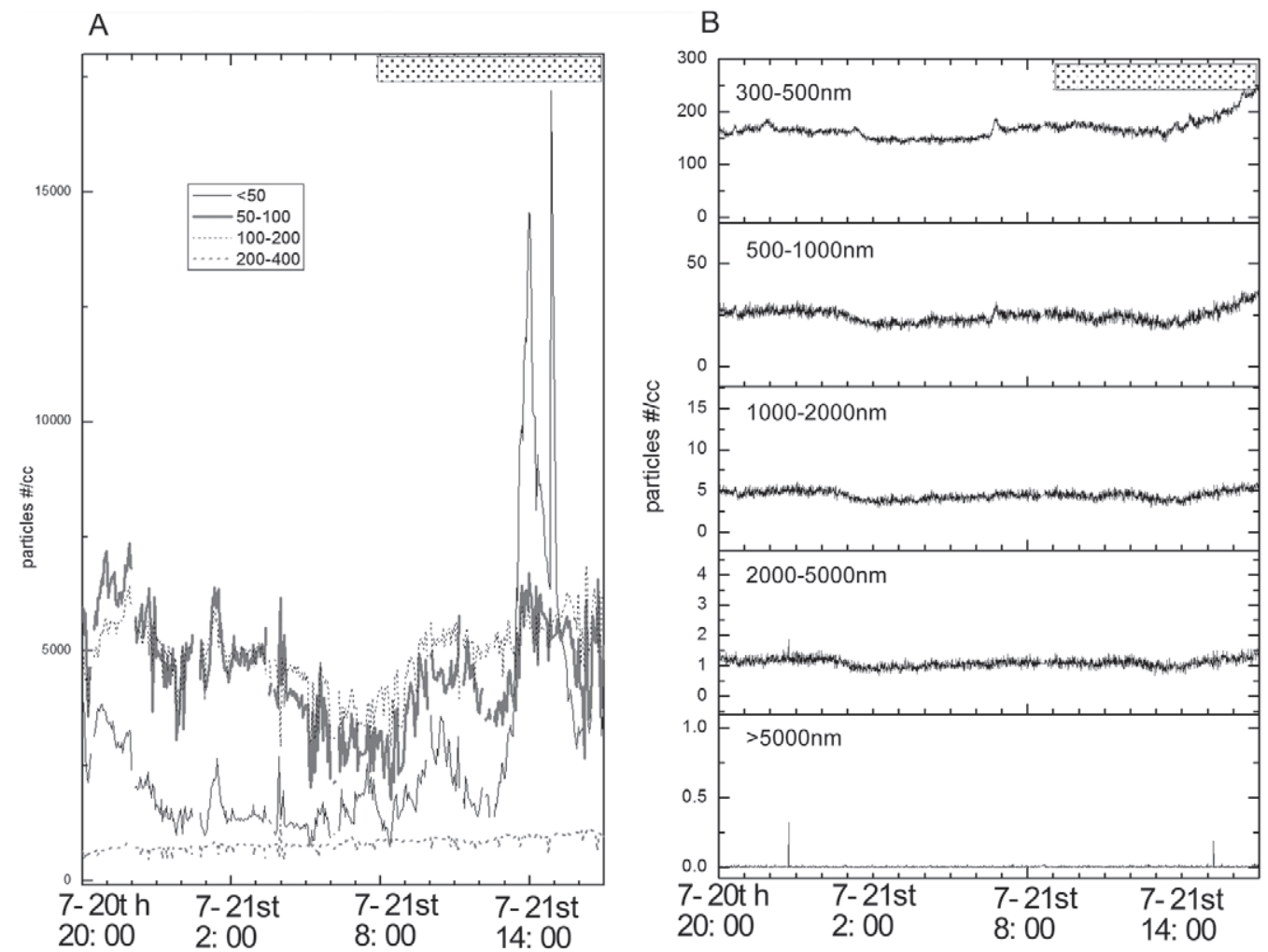

נr. with MWCNT coated yarn fabrication

Fig. 2. Airborne particle concentration with/without the MWCNT-coated yarn weaving process at sampling Point (1).

A: Nanosize particle concentrations measure by SMPS, B: Sub-micron and micron size particle concentrations measured by OPC. During the measurement period, polyester (without MWCNT) textiles were being woven on other looms.

optical microscopic observation. Sections in which the MWCNT-coated yarn was detected were sliced into ultrathin sections 50-80 nm thick. The ultra-thin sections were examined by TEM (FEI Tecnai $\mathrm{G}^{2}$ sprit, FEI, Hillsboro, OR, USA) at the acceleration voltage of $120 \mathrm{kV}$.

\section{Results}

\section{Particle concentration and size distribution}

The mass concentrations of total/respirable dusts collected by personal sampling near the operator were 0.092/0.066 $\mathrm{mg} / \mathrm{m}^{3}$ (total/respirable) at Point (1) (stationary sampling near the loom) and $0.159 / 0.093 \mathrm{mg} / \mathrm{m}^{3}$ (total $/$ respirable) near the operator (personal exposure). Although the samples for Point (1) and personal exposure had different positions, the dust mass concentrations were similar. The mass concentration of respirable particles measured using portable dustmonitor were 40 counts/min and 15 counts/min at Point (1) and in the office, respectively. These values corresponded to $0.040 \mathrm{mg} / \mathrm{m}^{3}$ and $0.002 \mathrm{mg} / \mathrm{m}^{3}$ by calculating with the value of mass-concentration coefficient, which is called " $\mathrm{K}$ coefficient" in Japan. In this case, we used the coefficient of 1,000 counts $/ \mathrm{min}=1 \mathrm{mg} / \mathrm{m}^{3}$. The K-coefficient is the same as that for polystyrene latex (PSL) particles, which were used as calibration particles. We used this value because we speculated that the most part of the dusts in the factory were comprised of polyester which is similar to PSL.

Figure 2 shows the number concentration of nanosize and submicron-size particles taken between the evening of July 20 and the evening of July 21; the MWCNTcoated yarn weaving process was carried out on July 21 with irregular suspensions to tune the loom. In terms of particle number concentrations, neither the nanosize nor submicron-size particles showed any task-related tendencies. The particle size distributions inside and outside the facility of weaving are shown in Fig. 3. From the similarity of the two curves, it is a reliable estimate that the origin of the nanoparticles in the weaving room was outside 


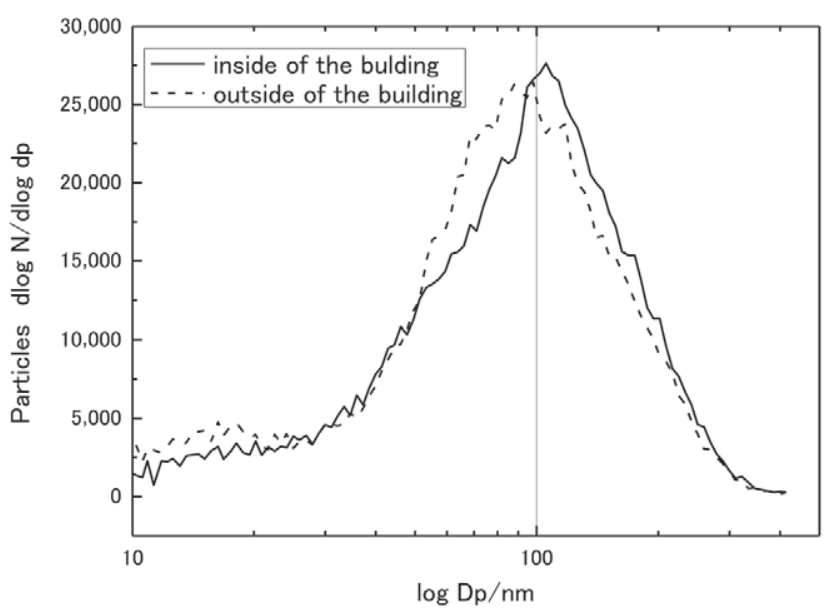

Fig. 3. Particle size distributions of nanosize particles in the air inside and outside the facility building during the MWCNT-coated yarn weaving process.

Solid line: the airborne dust in the building. Dotted line: the airborne dust outside the building. ambient air. Therefore, the nanosize particle concentration near the loom was influenced by the outside air rather than the weaving process.

\section{Particle characterization with SEM}

To confirm the presence of MWCNT fibers or MWCNTcontaining particles, SEM observation was conducted. Figure 4 shows SEM images of 6,600-2,500 nm particles in the air around the loom both with and without the MWCNT-coated yarn weaving process. No nanosize individual fibrous MWCNT particles were observed but many micron-size particles, which seemed to be fragments of yarn, were observed. Only in the sample collected during the weaving process were micron-size fibrous particles observed. The particles having nanosize fiber on their surface should be fragments of the MWCNT-coated yarn. Without the weaving process, only fragments of polyester yarn were observed. Therefore, we concluded the nanosize fibers on the surface of the micron-size fragments were MWCNT, and the fragmented particles with MWCNT were produced
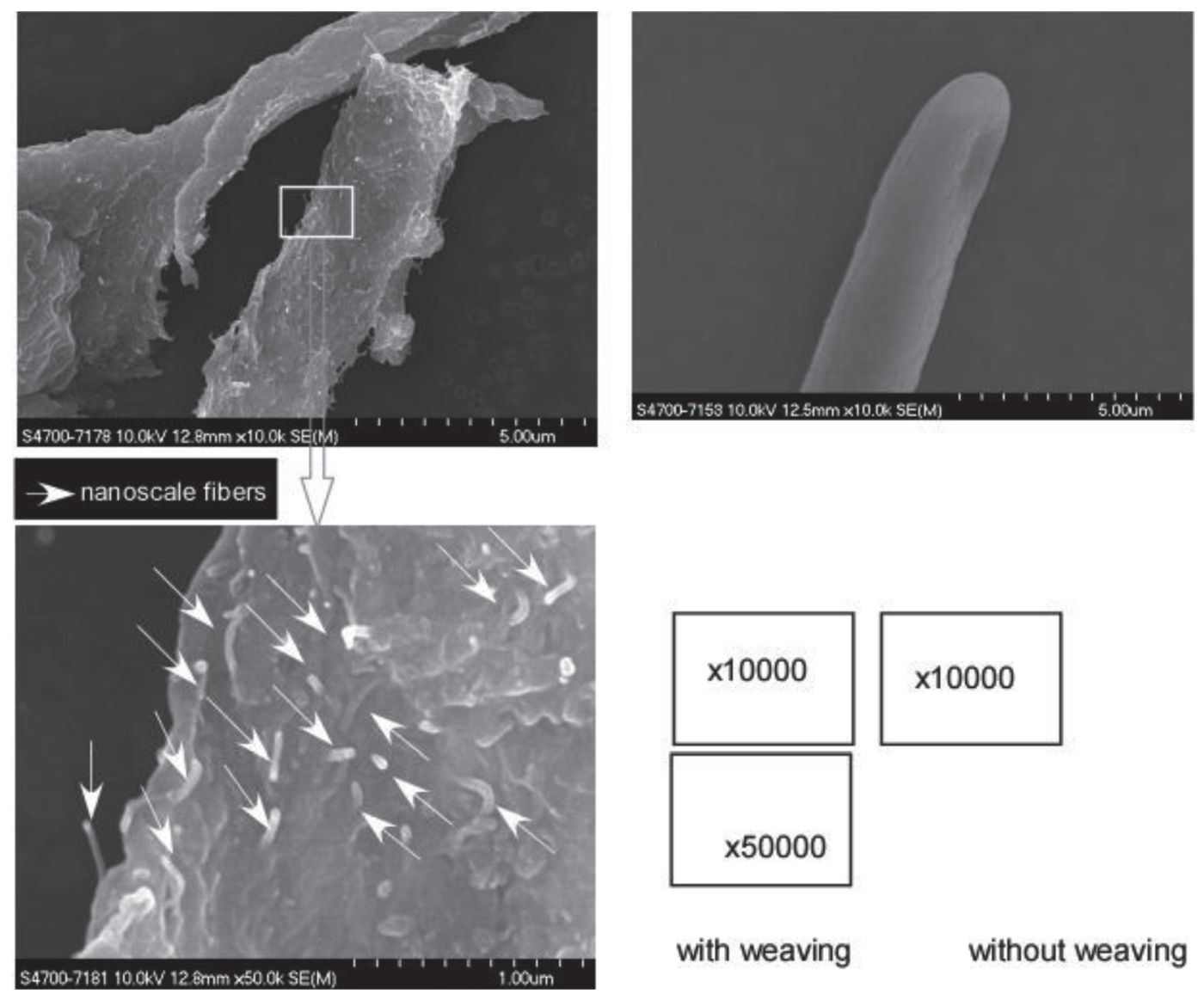

with weaving

Fig. 4. Scanning Electron Microscope (SEM) images of the dusts in the air around of the loom with/without the MWCNT-coated yarn weaving process. 


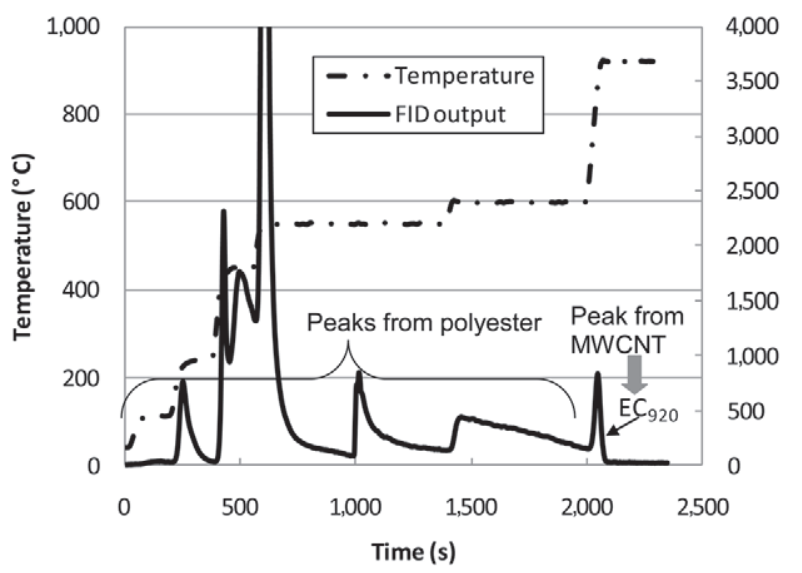

Fig. 5. Thermogram of CNTEC $(1.5 \mathrm{~cm})$.

by the mechanical forces of weaving. The particles classified as submicron-size by SCI sampling were considered to be primarily from outside air because of their morphology. No fibrous particles were observed in this size range.

\section{Carbon analysis}

Figure 5 shows a thermogram, an output chart of carbon analysis, of MWCNT-coated yarn. Polyester fiber and chemicals used for adhesion of MWCNT were oxidized at the temperature up to $600^{\circ} \mathrm{C}$. MWCNT was oxidized and detected as $\mathrm{EC}_{920}$. Table 2 shows the $\mathrm{EC}_{920}$ for particles collected at all stages of SCI. The $\mathrm{EC}_{920}$ values were lower when the weaving was not taking place, and much higher during the adjustment of the loom and frequent checks of the tension of the MWCNT-coated yarn in the morning. In this case, personal exposure data was that for a person standing 1 to $2 \mathrm{~m}$ away from the loom, not for the operator. Personal exposure data was at the same level as that of the environmental concentration of Point (1) and was also higher in the morning. The data for Point (2) close to the shed of the loom (the weft-delivering position with the rapier) was slightly higher than that of other data. Table 2 also shows the ratios of $\mathrm{EC}_{920}$ for particles larger than $1,000 \mathrm{~nm}$ to that for all sizes of particles. This ratio indicates the contribution of micron-size fragments to total particles with reference to the results of SEM observation. Higher ratios were observed during manual tasks and near the shed where a higher mechanical force is applied to the MWCNT-coated yarn. The ratio decreased in the afternoon because of a relatively higher concentration of submicron size ambient particles and the decreased frequency of manual operations. Although ambient particles and polyester yarns showed positive interference with the carbon analysis
Table 2. Concentrations of $\mathrm{EC}_{920}(=\mathrm{MWCNT})$ and ratios of $\mathrm{EC}_{920}$ in particles larger than $1,000 \mathrm{~nm}$ to all size

\begin{tabular}{lcc}
\hline Sample name & $\begin{array}{c}\mathrm{EC}_{920}(<6,600 \mathrm{~nm}) \\
\left(\mathrm{mg} / \mathrm{m}^{3}\right)\end{array}$ & \begin{tabular}{c}
$\mathrm{EC}_{920}(>1,000 \mathrm{~nm})$ \\
\hline 1 w/o Work
\end{tabular} $\mathrm{EC}_{920}($ Stages A-E $)$ \\
1 1-AM & 0.0011 & 0.21 \\
1 -PM & 0.0048 & 0.30 \\
1 and 2** & 0.0037 & 0.14 \\
Personal AM & 0.0053 & 0.58 \\
Personal PM & 0.0048 & 0.30 \\
\hline
\end{tabular}

of MWCNT, $\mathrm{EC}_{920}$ could be used as the index of MWCNTcontaining particles release. The concentrations of $\mathrm{EC}_{920}$ in this environment were lower than $0.0053 \mathrm{mg}-\mathrm{C} / \mathrm{m}^{3}$.

Wipe samples around the loom were also analyzed. For these samples, a clear and large single peak of $\mathrm{EC}_{920}$ was observed in the carbon analysis. The amounts of $\mathrm{EC}_{920}$ of samples on the shelf plate near reels and on the top of the loom were $0.005 \mathrm{mg}-\mathrm{C} / 100 \mathrm{~cm}^{2}$ and $0.003 \mathrm{mg}-\mathrm{C} / 100 \mathrm{~cm}^{2}$, respectively. This means that there were deposits of large numbers of fragments from the MWCNT-coated yarn near the site where a strong mechanical force is applied to the yarn.

\section{Sample yarns analysis by TEM}

Figure 6 shows the cross-sectional TEM images of an ultrathin-slice sections of the MWCNT-coated yarn. Under the coating layer of the yarn, there were tangled structures of tubular particles, which we considered were MWCNT fibers. Some individual MWCNT fibers were observed protruding from the coating. We considered that these fibers were more likely to be released into the air as individual MWCNT fibers; therefore, we tried counting the numbers of fibers protruding from the surfaces of the unwoven raw yarn and from yarn samples picked out from the woven textile. The counting criterion was a protruding length longer than $50 \mathrm{~nm}$. From the observation of approximately 120 images of the each type of sample, the geometric mean of the protruding MWCNT fibers were $5,035 / \mathrm{mm}$ on the raw yarn and $4,379 / \mathrm{mm}$ on the woven samples, respectively. Those values were compared using Student's $t$-test but they were not significantly different at the $5 \%$ level. Structure change of protruding fibers was not examined.

\section{Discussion}

We have proposed a systematic protocol for assessing the risk of exposure due to handling nanomaterials in the 


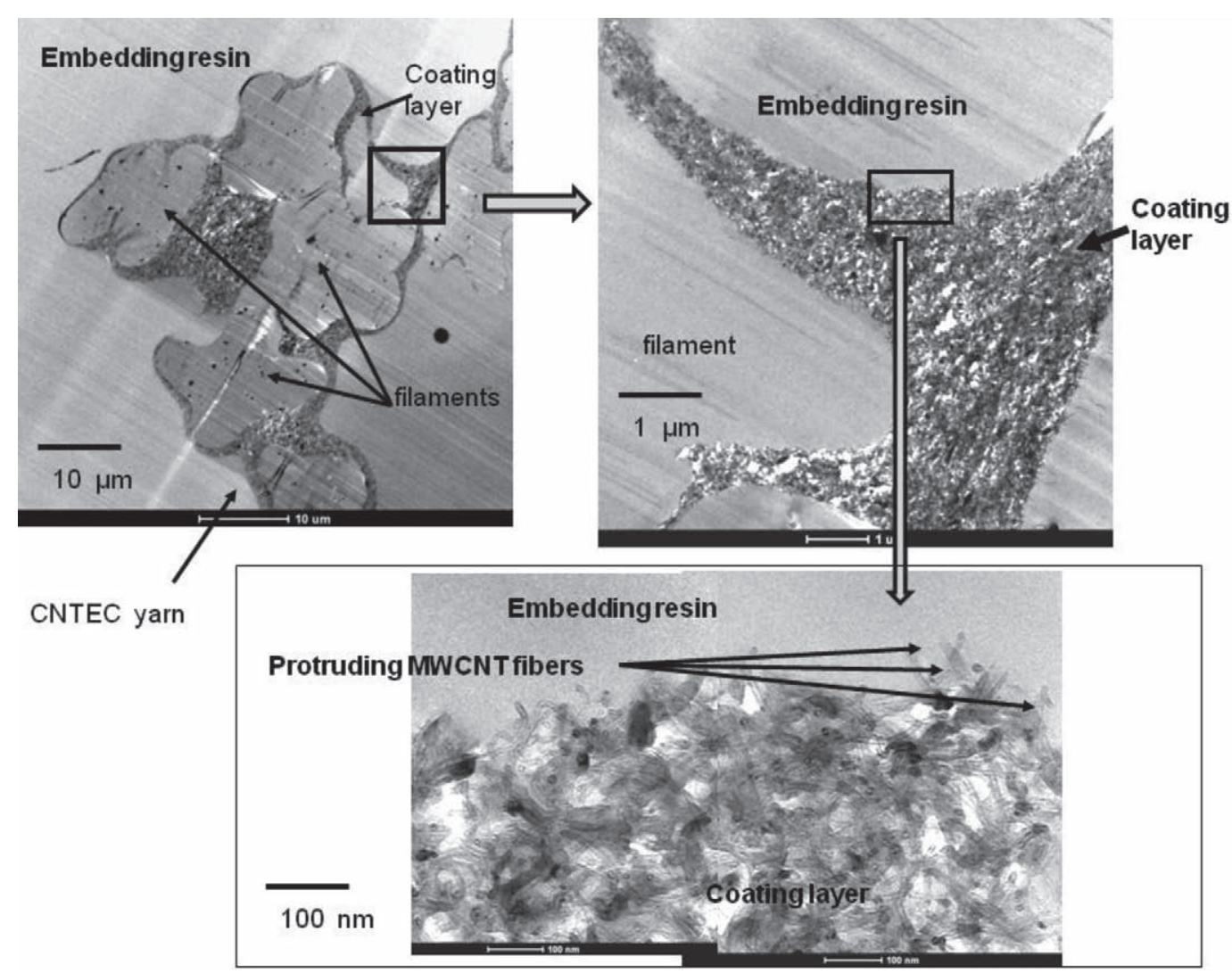

Fig. 6. Cross sectional images by Transmission Electron Microscopy (TEM) of the MWCNT-coated yarn. The tangled structures of fibrous tubular particles are the MWCNT in a coating layer.

workplace $^{12)}$. In the present study, the measurement and analytical procedure was based upon the protocol. The MWCNT assessment protocol in the workplace air was as follows: 1) real-time measurement, 2) electron microscopic observation, and 3) quantitative carbon analysis.

\section{Monitoring release of MWCNT containing dust particles} into the workplace

In the weaving process, the release of nanosize particles into the workplace air was not detected by the measurement of either particle concentration or size distribution. Hence, the concentration increase of work-related particles was masked by higher concentrations of background particles. Although the factory is located in a remote area, and thus it is rarely affected by combustion-derived particles, there must have been nanosize particles by secondary and photochemical generation from gaseous substances. The particle concentration of inside air was consistently lower than that of outside air during the survey period. In contrast, the micron-size particle concentration of the workplace air was higher than that of the outside air and the air in the office, which indicates that the weaving process caused some dust release. The major source of the particles was plain (non-MWCNT-treated polyester yarn, because MWCNT-coated yarn was woven on only one loom while over 20 looms were weaving plain yarn.

Because there was no detection of nanosize particles in real-time measurement, SEM observation and carbon analysis were required to assess the release of MWCNTrelatied particles.

In the SEM observation, micron-size particles with nanosize fibers on their surfaces were found. It is likely that the particles were fragments of the MWCNT-coated yarn and/or MWCNT coating broken by the mechanical force of the weaving. Individual MWCNT particles were not found under the SEM observations. The results show that the strength of the mechanical force was enough to break the coating; however, it was insufficient to release MWCNT from the coating layer.

Because SEM observation is not suitable for quantitative analysis, the collected particles were also analyzed with carbon analysis to determine the MWCNT concentrations. The estimated MWCNT concentration of the particles under 6,600 nm was not higher than 0.0053 
$\mathrm{mg}-\mathrm{C} / \mathrm{m}^{3}$. The concentration was relatively high during the MWCNT-coated-yarn weaving, especially during manual tuning tasks. Also, the samples collected near the yarn under tension showed higher MWCNT concentrations.

\section{Possibility of release of individual nanosize MWCNT fiber into the workplace}

To evaluate the release of individual MWCNT fibers from the MWCNT-coated yarn into the workplace, a cross-sectional observation of the MWCNT-coated yarn using TEM was conducted. There are no established evaluation criteria for assessing the release possibilities of individual fibers. In the present study, we hypothesized that the tubular fibers sticking out from the surface of the yarn that were longer than $50 \mathrm{~nm}$ had a higher probability of fall-off. Accordingly, we counted the number of tubular fibers under the $50-\mathrm{nm}$ criterion, the number did not significantly change between before and after the weaving process. Taken together with the other results, we consider the possibility of release of individual MWCNT fibers into the workplace in the weaving process was low.

\section{Summary}

During the weaving process of the MWCNT-coated yarn, there were some micron-size particles containing MWCNT that were released into the air around the loom, probably by the mechanical forces applied to the yarn. Even though nanosize MWCNT particles were not found in this case, we recommend that workers use conventional (not specialized for nanoparticles) personal protection equipment such as respirators and gloves to mitigate exposure, because of the existence of respirable-size MWCNT-containing particles.

\section{Acknowledgements}

We are deeply grateful to Kuraray Living Co., Ltd. and Matsubun Textile Co. Ltd. for giving us the important opportunity to conduct a field survey in a MWCNT-related textile factory. We are also deeply indebted to Dr. Isamu Ogura of the National Institute of Advanced Industrial Science and Technology (AIST) for his advice given in fruitful discussions about the present study.

\section{References}

1) Fugetsu B, Han W, Endo N, Kamiyama Y, Okuhara T (2005) Disassembling single-walled carbon nanotube bundles by dipole/dipole electrostatic interactions. Chem Lett 34,
1218-9.

2) Patent application 2007-225966, 2008-224821.

3) Development of novel electro-conductive fiber by carbon nano-tubes coating (2010) Report of "Regional Innovation Creation R\&D Programs in FY2009”, Aichi Science \& Technology Foundation, Aichi (in Japanese).

4) Bayer Material Science (2009). http://www.baytubes.com/ news_and_services/news_091126_oel.html. Accessed July 12, 2011.

5) Draft version: Occupational Exposure to NIOSH current intelligent bulletin: Occupational Exposure to Carbon Nanotubes and Nanofibers (2010) Centers for Disease Control and Prevention, National Institute for Occupational Safety and Health NIOSH DHHS (NIOSH) Publication No. 2010-XXX; Date: December 2010. National Institute for Occupational Safety and Health, Cincinnati. http:// www.cdc.gov/niosh/docket/review/docket161A/pdfs/ carbonNanotubeCIB_PublicReviewOfDraft.pdf. Accessed July 12, 2011.

6) Kobayashi N, Ogura I, Gamo M, Kishimoto A, Nakanishi $\mathrm{J}$ (2009) Executive summaries of interim reports on risk assessments of three manufactured nanomaterials: carbon nanotubes (CNTs), (issued on October 16, 2009): The Research Institute of Science for Safety and Sustainability, National Institute of Advanced Industrial Science and Technology (AIST), Tsukuba.

7) Han JH, Lee EJ, Lee JH, So KP, Lee YH, Bae GN, Lee S-B, Ji JH, Cho MH, Yu IJ (2008) Monitoring multiwalled carbon nanotube exposure in carbon nanotube research facility. Inhal Toxicol 20, 741-9.

8) Takaya M, Serita F, Ono-Ogasawara M, Shinohara Y, Saito H, Koda S (2010) Airborne particles in a multi-wall carbon nanotube production plant: observation of particle emission and personal exposure 1: measurement in the packing process. San Ei Shi 52, 182-8 (in Japanese).

9) Methner M, Hodson L, Dames A, Geraci C (2010) Nanoparticle emission assessment technique (NEAT) for the identification and measurement of potential inhalation exposure to engineered nanomaterials_-Part B: Results from 12 field studies. J Occup Environ Hyg 7, 163-76.

10) Wohlleben W, Brill S, Meiser MW, Mertler M, Cox G, Hirth S, von Vacano B, Strauss V, Treumann S, Wiench K, Ma-Hock L, Landsiedel R (2011) Small, DOI: 10.1002/ smll.201002054.

11) Ono-Ogasawara M, Serita F, Takaya M (2009) Distinction between nanomaterial particle and unintentional airborne particulate matter. J Nanopart Res 11, 1651-9.

12) Misra C, Singh M, Shen S, Sioutas C, Hall PM (2002) Development and evaluation of a personal cascade impactor sampler (PCIS). J Aerosol Sci 33, 1027-47.

13) Chow JC, Watson JG, Pritchett LC, Pierson WR, Frazier CA, Purcell RG (1993) The DRI thermal/optical reflectance carbon analysis system: description, evaluation and applications in U.S. Air quality studies. Atmos Environ 27A, 1185-201. 